\title{
Comparing universal Lynch syndrome tumor-screening programs to evaluate associations between implementation strategies and patient follow-through
}

\author{
Deborah Cragun, MS, PhD'1, Rita D. DeBate, $\mathrm{PhD}^{2}$, Susan T. Vadaparampil, $\mathrm{PhD}^{1}$, Julie Baldwin, $\mathrm{PhD}^{2}$, \\ Heather Hampel, $\mathrm{MS}^{3}$ and Tuya Pal, MD ${ }^{1}$
}

\begin{abstract}
Purpose: Universal tumor screening (UTS) for all colorectal cancer patients can improve the identification of Lynch syndrome, the most common cause of hereditary colorectal cancer. This multiple-case study explored how variability in UTS procedures influenced patient follow-through $(\mathrm{PF})$ with germ-line testing after a screen-positive result.
\end{abstract}

Methods: Data were obtained through Web-based surveys and telephone interviews with institutional informants. Institutions were categorized as Low-PF ( $\leq 10 \%$ underwent germ-line testing), Medium-PF (11-40\%), or High-PF (>40\%). To identify implementation procedures (i.e., conditions) unique to High-PF institutions, qualitative comparative analysis was performed.

Results: Twenty-one informants from 15 institutions completed surveys and/or interviews. Conditions present among all five High-PF institutions included the following: (i) disclosure of screen-positive results to patients by genetic counselors; and (ii) genetic counselors either facilitate physician referrals to genetics professionals or eliminate the need for referrals. Although both of these High-PF conditions were present among two Medium-PF institutions, automatic reflex testing was lacking and difficulty contacting screen-positive patients was a barrier. The three remaining Medium-PF and five Low-PF institutions lacked the conditions found in High-PF institutions.

Conclusion: Methods for streamlining UTS procedures, incorporating a high level of involvement of genetic counselors in tracking and communication of results and in reducing barriers to patient contact, are reviewed within a broader discussion on maximizing the effectiveness and public health impact of UTS.

Genet Med advance online publication 20 March 2014

Key Words: hereditary colorectal cancer; Lynch syndrome; Public Health Genomics; qualitative comparative analysis; RE-AIM

\section{INTRODUCTION}

Colorectal cancer (CRC) is the second leading cause of cancer-related death in the United States when men and women are considered together. ${ }^{1}$ Lynch syndrome is the most common cause of hereditary CRC, affecting $\sim 1$ in every 35 CRC patients. ${ }^{2}$ Individuals with Lynch syndrome have a 50-70\% lifetime risk of CRC, ${ }^{3-5}$ a $40-60 \%$ chance of endometrial cancer (in women), ${ }^{3,6}$ and increased risks for several other malignancies. ${ }^{6,7}$ Fortunately, effective strategies for cancer risk reduction are available when Lynch syndrome is identified. ${ }^{8,9}$

The importance of Lynch syndrome identification is reflected in the following Healthy People 2020 provisional objective: "Increase the proportion of persons with newly diagnosed colorectal cancer who receive genetic testing to identify Lynch syndrome." Systematic efforts to identify patients with Lynch syndrome are needed given the current estimate that less than $5 \%$ of individuals with Lynch syndrome have been diagnosed. ${ }^{10,11}$ Relying on age or family history criteria to determine eligibility for Lynch syndrome screening or testing misses between 25 and $70 \%$ of Lynch syndrome patients. ${ }^{12-16}$ Therefore, several institutions are now adopting a universal tumor screening (UTS) approach to determine which patients should be offered genetic counseling and germ-line testing for Lynch syndrome..$^{17,18}$

UTS programs are endorsed by the Centers for Disease Control and Prevention Office of Public Health Genomics on the basis of evidence of analytic validity, clinical validity, and clinical utility. ${ }^{19-21}$ In addition, economic models have found the costs of UTS to be comparable to the costs of other preventive services adopted within the United States. ${ }^{22,23}$ Furthermore, a large private health-care system has implemented UTS after independently weighing costs and benefits. ${ }^{24}$

UTS procedures are known to vary across institutions. ${ }^{17,18}$ Laboratory procedures for UTS include microsatellite instability testing and/or immunohistochemical testing to identify tumor mismatch repair deficiency. In the case of a subset of mismatch repair-deficient tumors, reflex BRAF V600E mutation and/or hypermethylation testing may be added to rule out patients who are unlikely to have Lynch syndrome. ${ }^{18,20,25}$ Variations in results follow-up procedures include different methods for tracking and disclosing results. An additional procedural consideration is whether patients' informed consent is

\footnotetext{
${ }^{1}$ Division of Population Science, Moffitt Cancer Center, Tampa, Florida, USA; ${ }^{2}$ Department of Community and Family Health, University of South Florida, Tampa, Florida, USA;

${ }^{3}$ Department of Internal Medicine, Ohio State University, Columbus, Ohio, USA. Correspondence: Deborah Cragun (deborah.cragun@moffitt.org) 
obtained before screening or whether screening is implemented as part of standard procedure.

Regardless of the chosen procedures, the clinical benefits of UTS can be realized only if a high proportion of screen-positive patients (i.e., with results that suggest possible Lynch syndrome) follow through with genetic counseling and germ-line testing to confirm a diagnosis and obtain recommendations and options to prevent future cancers for themselves and their at-risk relatives. This multiple-case study compared UTS adoption, implementation, and effectiveness across several existing UTS programs. The objectives of the study were to (i) identify challenges and facilitators to UTS adoption; (ii) further characterize similarities and differences in UTS procedures that have been implemented at different institutions; (iii) identify suboptimal outcomes of UTS; and (iv) develop a model to explain varying levels of patient follow-through $(\mathrm{PF})$ with germ-line testing across institutions.

\section{MATERIALS AND METHODS Study Frameworks}

Two complementary frameworks, RE-AIM (Reach, Effectiveness, Adoption, Implementation, Maintenance) ${ }^{26-28}$ and the Consolidated Framework for Implementation Research, ${ }^{29}$ were used in planning of the study and in the design of surveys and interview guides. The use of RE-AIM was expected to increase the quality, speed, and public health impact of stakeholder efforts to more effectively translate UTS into practice by considering the following five dimensions ${ }^{26-28}$ : (i) Reach-the absolute number, proportion, and representativeness of CRC patients who are screened for Lynch syndrome; (ii) effectiveness-the impact of UTS procedures on PF and other outcomes, including potential negative effects; (iii) adoption-the absolute number, proportion, and representativeness of institutions and staff who adopt UTS, in addition to the resources and expertise available to them; (iv) implementation - time and costs of UTS programs, and what adaptations are made to UTS in various settings; and (v) maintenance - the extent to which UTS becomes part of routine practice and the effects of UTS over time. The Consolidated Framework for Implementation Research, which is described in more detail in Supplementary Table S1 online, was used because it incorporates specific factors that may influence the decision to adopt an innovation (i.e., UTS), and it includes several implementation conditions that can influence overall effectiveness. ${ }^{29}$

\section{Study Design}

Following institutional review board approval of this multiplecase study, key informants at institutions performing UTS completed an initial survey. Follow-up surveys and interviews were then conducted 6 months later to obtain maintenance data and to inform the interpretation of earlier findings.

\section{Participant Recruitment, Study Procedures, and Measures}

Sampling frame. By June 2012, the membership of the Lynch Syndrome Screening Network (LSSN) consisted of more than 70 institutions across the United States. Of these, 35 were actively screening all newly diagnosed CRC patients regardless of age or other factors (i.e., they had adopted UTS). Given the current study's focus on system-level implementation rather than patient-level influences, institutions that were not performing UTS were excluded to reduce variation among the patient populations screened at different institutions.

Initial survey of key informants. LSSN representatives from institutions performing UTS were eligible and were invited to participate in the initial survey via an e-mail invitation posted twice within a 2-month period on the LSSN listserv. From midOctober through December 2012, interested representatives served as key informants by completing an online survey collecting information on the following: (i) institutional characteristics; (ii) factors influencing the decision to adopt UTS; (iii) challenges and facilitators to UTS adoption; (iv) UTS procedures; (v) the percentage of patients who follow through with germ-line testing after a positive screen (i.e., PF); and (vi) barriers or facilitators to PF. Before study initiation, the survey was reviewed for face and content validity by a medical geneticist, two genetic counselors (GCs), an epidemiologist, and a behavioral scientist specializing in cancer. The revised survey was piloted by two GCs and a nurse practitioner involved in UTS programs. The final online survey included five openended questions and $\sim 20$ multipart, closed-ended questions with an option to write in other responses.

Six-month follow-up with institutions. Key informants were e-mailed 1-2 requests asking them to provide additional information in all cases in which the institution met the following more stringent inclusion criteria: (i) UTS had been fully implemented for 6 months or longer at the time of the initial survey; and (ii) data on PF were available. To obtain additional UTS details, clarify information, and identify changes in UTS procedures, follow-up surveys and/or interviews were conducted with key informants and secondary informants. Surveys and semi-structured interview guides consisted of closed- and openended questions that had been reviewed for face and content validity by several specialists familiar with UTS. Although there was some overlap with questions from the initial survey, most questions included in follow-up surveys and interviews were designed to obtain additional UTS details, clarify information, and identify changes in UTS procedures. All interviews were audio recorded and notes were taken by the interviewer.

Primary outcome and conditions. The initial and followup surveys contained a question asking for the approximate percentage of patients with a screen-positive result who pursue germ-line testing. Response options were the following: $1=\leq 10 \% ; 2=11-25 \% ; 3=26-40 \% ; 4=41-55 \% ; 5=56-70 \%$; $6=71-85 \%$; and $7=>85 \%$. Ordinal responses to this question from the initial survey were used as the primary outcome (i.e., PF score). Other questions from the initial survey measured the presence of implementation conditions hypothesized to influence the outcome. These questions are included in Table 1, 
Table 1 Sample of select study questions and responses, along with their relation to RE-AIM dimensions and/or constructs from the Consolidated Framework for Implementation Research

\section{Initial survey questions \\ Response options}

Approximately how many colorectal cancer patients have been screened at your institution in the past 6 months (or less if you have recently implemented UTS)?

What were the primary reasons for implementing UTS at your institution? (check all that apply)

- UTS was recommended in 2009 by the Evaluation of Genomic Applications in Practice and Prevention (EGAPP) Working Group

- To improve the identification of patients with Lynch syndrome

- To reduce cancer mortality

- To benefit relatives of individuals with Lynch syndrome

- To generate increased revenue

- To "keep up" with other institutions that were already performing UTS

What barriers (if any) did your institution face when planning UTS? (check all that apply)

- No real barriers

- Difficulty in convincing key stakeholders (e.g., administrators and health-care providers) why UTS was important

- Arranging time when individuals could meet to discuss UTS was challenging

- Lack of laboratory expertise/resources

- Concerns were raised regarding the need for informed consent

- Difficulty deciding which screening method to use (i.e., IHC versus MSI)

- Disagreement occurred about how results should be handled

- Concerns about the cost of screening were raised

- One or more individuals tried to prevent UTS implementation

- Communication barriers existed between key stakeholders (e.g., administrators, pathologists, and health-care providers)

- Perceptions that other screening algorithms are more cost effective or superior to UTS

What factors were helpful when planning and implementing UTS at your institution? (check all that apply)

- Prior to UTS, my institution was already routinely screening a subset of colorectal tumors

- An "institutional champion" worked hard to implement UTS

- A high-level administrator or supervisor supported UTS

- Collaborative relationships existed across departments and/or working groups

- Protected time was provided for planning UTS

- Useful information was obtained from another institution that was already performing UTS

- Multiple planning meetings helped facilitate UTS implementation

Who is responsible for disclosing abnormal screening results to the patients?

How are abnormal screening results usually disclosed to patients? (e.g., phone, in person)

What do you think may be preventing some colorectal cancer patients from receiving germ-line testing after an abnormal tumor screen at your institution? (check all that apply)

- Lack of adequate insurance coverage and/or financial difficulties

- Health-care providers fail to recognize the need for germ-line testing

- Referral to genetics by other health-care provider is required

- Inconvenient for patients to arrange and/or attend a separate genetics appointment

- Difficulty in contacting patients to set up germ-line testing

- Lack of patient understanding about the importance of genetic counseling

- Patients are dealing with too many other issues at the time of diagnosis

- Patients don't want to face the possibility that others in their family may be at increased risk for cancer

- Patients are concerned about genetic discrimination

Of those colorectal cancer patients who have an abnormal tumor screen, approximately what percentage do you think provide a blood or saliva sample for germ-line testing?

How often have patients at your institution expressed concerns or responded negatively to tumor screening? Has your institution experienced any problems or unanticipated outcomes related to universal colorectal tumor screening?

\section{RE-AIM dimension}

CFIR construct

Reach

Adoption

External policy

Relative advantages

Peer pressure

Adoption

Not applicable

Knowledge and beliefs

Resources

Patient needs

Complexity and planning

Cost

Engagement

Communication

Relative advantage

Adoption

Trialability

Engagement

Networks and communication

Resources

Access to information

Planning

Execution

Execution

Effectiveness

Patient resources

Beliefs and knowledge

Execution

Patient needs

Execution

Not applicable

Not applicable

Not applicable

Not applicable

Effectiveness

Effectiveness

Effectiveness

Follow-up survey questions

How often does someone meet the patient at a routine follow-up visit (i.e., surgical follow-up) to discuss germ-line Execution testing when indicated (Likert-type scale for response) 


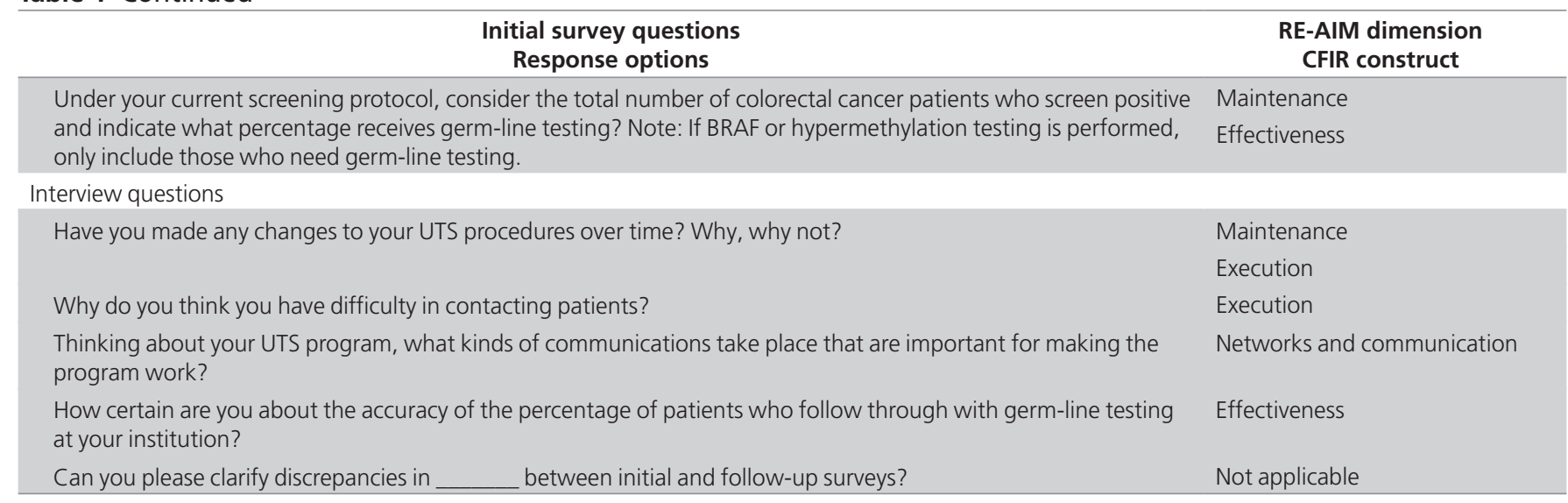

Questions on both the initial and follow-up surveys asked several other detailed questions about how UTS procedures are executed.

CFIR, Consolidated Framework for Implementation Research; IHC, immunohistochemical testing ; MSI, microsatellite instability testing; RE-AIM, Reach, Effectiveness, Adoption, Implementation, Maintenance; UTS, universal tumor screening.

along with select questions from follow-up surveys and interviews that aided in interpretation of results.

\section{Data Analysis}

Descriptives. After arranging the institutions in descending order by $\mathrm{PF}$ scores, institutions were categorized into three groups: High-PF ( $>40 \%)$; Medium-PF (11-40\%), and Low$\mathrm{PF}(\leq 10 \%)$. Frequencies and percentages were generated for responses to closed-ended survey questions. Open-ended responses and interview data were reviewed to identify commonalities and diversity across institutions and to characterize each RE-AIM dimension.

Implementation procedures associated with PF. Qualitative comparative analysis (QCA) is an analytic technique for performing cross-case comparisons to identify conditions that are "sufficient" for an outcome of interest to occur. 30,31 Although QCA is quite different from inferential statistics, conditions (i.e., implementation procedures) are analogous to independent variables hypothesized to influence the outcome of interest (i.e., PF). Using QCA, combinations of conditions uniquely associated with high- and low-PF were determined.

Before conducting QCA, conditions were coded as $1=$ condition present; $0=$ condition absent. $\mathrm{PF}$ was coded into two different variables for two separate QCA analyses: (i) High-PF $=$ 1 if institutional PF score was $>40 \%$ and High-PF $=0$ for all other institutions; (ii) Low-PF $=1$ if PF score was $\leq 10 \%$ and Low-PF $=0$ for all others. Specialized software (fsQCA 2.0) and the truth table approach ${ }^{32}$ were used to identify combinations of conditions unique to High-PF institutions and those unique to Low-PF institutions. Steps used to perform QCA are outlined in Supplementary Table S2 online. QCA solutions were obtained from Boolean simplification of the data matrix shown in Table 2; solutions were then triangulated with 6-month follow-up data to formulate mechanistic models by which these conditions may influence PF.

\section{RESULTS}

Respondent and Institution Characteristics

Of 35 LSSN representatives at institutions performing UTS, 20 (57\%) completed the initial survey, of which 15 institutions met stringent inclusion criteria for cross-case comparisons. All key informants were GCs, except for one informant from an institution not meeting inclusion criteria. Additional information was collected at 6-month follow-up via interviews and/or follow-up surveys completed by 12 of the 15 key informants whose institutions met inclusion criteria. Interviews were also completed with six secondary informants from 4 of the 15 institutions. Table 3 provides characteristics of all participating institutions, including the five for which PF data were not available. Table 3 also presents institutional characteristics according to PF. Four of the five High-PF institutions were National Cancer Institutedesignated academic/research institutions. By contrast, three of the five Medium-PF and three of the five Low-PF institutions were nonacademic/nonresearch institutions. Among the 15 institutions meeting stringent inclusion criteria, 11 had been performing UTS for more than 1 year as of October 2012.

\section{RE-AIM}

Study findings within each of the five RE-AIM dimensions are presented in Table 4 and are summarized in the following sections. Table 4 also highlights the differences between High-PF and Low-PF institutions.

Patient reach. Estimated numbers of newly diagnosed CRC patients undergoing tumor screening are listed in Table 3. Although the proportion of all newly diagnosed CRC patients screened was not assessed, it was believed to be nearly $100 \%$ at all participating institutions based on the following: (i) institutional procedures dictated that tumors from all newly diagnosed CRC patients were to be screened; and (ii) no patients were reported to have opted out of screening. Nevertheless, it is possible that some tumors were missed.

Effectiveness. PF with germ-line testing after a positive tumor screen varied widely (ranging from $<10 \%$ to $>85 \%$ ) across 
Table 2 Data matrix of patient follow-through (PF) with germ-line genetic testing after a positive screen and conditions showing patterns associated with high- and low-PF

\begin{tabular}{|c|c|c|c|c|c|c|c|c|c|}
\hline \multicolumn{3}{|c|}{ Outcome $^{a}$} & \multicolumn{6}{|c|}{ Conditions $^{a}$} & \multirow{2}{*}{$\begin{array}{c}\begin{array}{c}\text { Intermediary } \\
\text { step }\end{array} \\
\\
\text { Follow- } \\
\text { through } \\
\text { with genetic } \\
\text { counseling }\end{array}$} \\
\hline $\begin{array}{l}\text { PF } \\
\text { genetic } \\
\text { testing }\end{array}$ & $\begin{array}{l}\text { High- } \\
\text { PF }\end{array}$ & $\begin{array}{l}\text { Low- } \\
\text { PF }\end{array}$ & $\begin{array}{l}\text { Challenges } \\
\text { to adoption } \\
\geq \text { facilitators }\end{array}$ & $\begin{array}{l}\text { Automatic reflex test } \\
\text { of screen-positive } \\
\text { tumors (e.g., BRAF) }\end{array}$ & $\begin{array}{l}\mathrm{GC}^{\mathrm{c}} \\
\text { receives } \\
\text { positive } \\
\text { screen } \\
\text { results }\end{array}$ & $\begin{array}{l}\text { GCc } \\
\text { discloses } \\
\text { screening } \\
\text { result to } \\
\text { patient }\end{array}$ & $\begin{array}{l}\text { Difficulty in } \\
\text { contacting } \\
\text { patients }\end{array}$ & $\begin{array}{l}\text { Need for } \\
\text { physician } \\
\text { referral is } \\
\text { a barrier }\end{array}$ & \\
\hline$>85 \%$ & 1 & 0 & 0 & 1 & 1 & 1 & 0 & 0 & $41-55 \%$ \\
\hline $71-85 \%$ & 1 & 0 & 0 & 1 & 1 & 1 & 0 & 0 & $71-85 \%$ \\
\hline $56-70 \%$ & 1 & 0 & 0 & 1 & 1 & 1 & 0 & 0 & $56-70 \%$ \\
\hline $41-55 \%$ & 1 & 0 & 0 & 1 & 1 & 1 & 0 & 0 & $>85 \%$ \\
\hline $41-55 \%$ & 1 & 0 & 1 & 1 & 1 & 1 & 0 & 0 & $71-85 \%$ \\
\hline $26-40 \%$ & 0 & 0 & 0 & 0 & 1 & 1 & 1 & 0 & $56-70 \%$ \\
\hline $26-40 \%$ & 0 & 0 & 0 & 1 & 1 & 0 & 1 & 1 & $41-55 \%$ \\
\hline $26-40 \%$ & 0 & 0 & 0 & 0 & 1 & 1 & 1 & 0 & $26-40 \%$ \\
\hline $26-40 \%$ & 0 & 0 & 0 & 1 & 1 & 0 & 0 & 1 & $11-25 \%$ \\
\hline $11-25 \%$ & 0 & 0 & 0 & 1 & 1 & 0 & 0 & 1 & $41-55 \%$ \\
\hline$\leq 10 \%$ & 0 & 1 & 1 & 0 & 1 & 0 & 0 & 1 & $11-25 \%$ \\
\hline$\leq 10 \%$ & 0 & 1 & 1 & 0 & 1 & 0 & 0 & 1 & $11-25 \%$ \\
\hline$\leq 10 \%$ & 0 & 1 & 1 & 1 & 1 & 0 & 0 & 1 & $\leq 10 \%$ \\
\hline$\leq 10 \%$ & 0 & 1 & 0 & 1 & 0 & 0 & 0 & 1 & $\leq 10 \%$ \\
\hline$\leq 10 \%$ & 0 & 1 & 0 & 0 & 0 & 0 & 0 & 1 & $\leq 10 \%$ \\
\hline
\end{tabular}

aThe presence of each outcome or condition used in qualitative comparative analysis is indicated with " $1 ;$ " the absence of each condition used in qualitative comparative analysis is indicated with " 0 ." bPF was determined from ordinal response options to a question on the initial survey asking about the percentage of patients who follow through with germ-line testing after a positive screen suggestive of Lynch syndrome. Responses ranged from $1(<10 \%)$ to 7 ( $>85 \%)$. . "GC" refers to a master's degreetrained genetic counselor or a nurse with extensive genetic counseling experience. ${ }^{\top} T h e$ percentage of patients who follow through with genetic counseling was asked (without specifying whether or not it had to be performed by a GC). Response options were the same as those for PF. Responses to this question were ultimately not used as part of the outcome or as a condition because genetic counseling could be considered an intermediary step.

institutions (Table 2). Negative outcomes related to UTS were uncommon, but included the following: (i) two patients who were unaware that tumor screening was part of their surgical informed consent; (ii) a few patients who were concerned about their inability to pay for genetic counseling and/or germ-line testing; (iii) the need to plan for handling results of testing on prison inmates or deceased patients; (iv) rare problems with reimbursement for tumor screening at two institutions; (v) one patient who, despite lack of interest, felt obligated to undergo germ-line testing; (vi) difficulties deciding how to follow up when results are equivocal or atypical; and (vii) concerns that physicians were not always disclosing screening results to patients.

Adoption. GCs were the first to propose the idea of UTS at most institutions, but pathologists, surgeons, gastroenterologists, or oncologists were typically very important in the decision to adopt UTS. At nonacademic institutions, administrators were often highly involved in decision making but were less so at academic institutions. The most commonly cited facilitators to UTS adoption were collaborative relationships that existed across departments, guidance from other institutions performing UTS, and having an institutional champion who supported UTS. Commonly cited challenges included concerns about whether active informed consent of the patient was necessary and concerns about screening costs or reimbursement. Additional challenges, primarily reported among Low-PF and nonacademic institutions, included (i) difficulty in convincing key stakeholders (e.g., administrators, health-care providers, and pathologists) why UTS is important; (ii) general lack of knowledge by stakeholders; and (iii) communication barriers between stakeholders.

Implementation and PF. The QCA results, reported in Table 4, revealed that all High-PF institutions share a combination of implementation conditions. High-PF institutions perform automatic reflex testing on a subset of screen-positive patients. In addition, High-PF institutions either do not require screen-positive patients to be referred for genetic counseling or GCs contact physicians to request and assist in completing referrals. At all High-PF institutions, patient disclosure of positive screening results is performed by a master's degree-trained GC or a genetic nurse counselor on behalf of the treating physicians. Although GCs routinely disclosed screening results to patients at two Medium-PF institutions, these GCs reported difficulty in contacting patients. By contrast, difficulty in contacting patients was not selected as a barrier by informants from High-PF institutions; in fact, three of these informants indicated that this barrier was overcome by having a GC or nurse meet the patient at an already scheduled follow-up appointment (e.g., surgical postoperative appointment). Interview data revealed that this approach is not feasible at some institutions 
Table 3 Characteristics of institutions and their respective universal tumor-screening programs

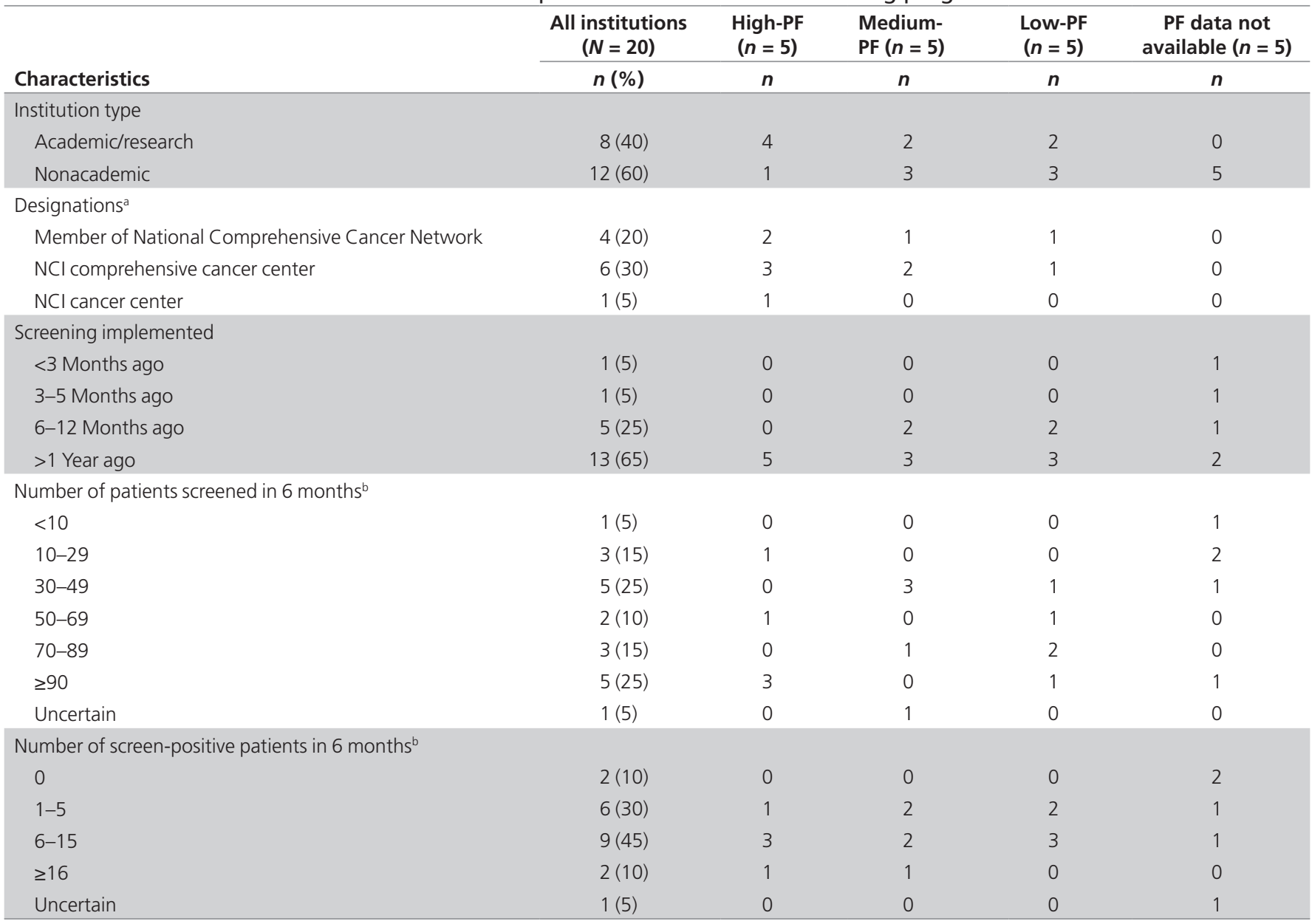

$\mathrm{NCl}$, National Cancer Institute; PF, patient follow-through with germ-line testing.

${ }^{a}$ National Comprehensive Cancer Network and NCl designations are independent, and some centers have both designations. ${ }^{b}$ Number within the past 6 months, or less if screening was implemented less than 6 months ago.

due to a lack of time among genetics personnel or because follow-up appointments occur at several different locations that are not in close proximity to GCs (i.e., private practices). In fact, physical distance was reported as the impetus for having a genetics nurse disclose positive screening results during postoperative appointments at one High-PF institution.

As shown in Table 4, two different sets of conditions distinguished Low-PF institutions from other institutions. Among all three nonacademic Low-PF institutions, a higher proportion of adoption challenges to facilitators was reported. At both of the academic Low-PF institutions, GCs did not receive detailed information on screen-positive patients.

Maintenance and PF. The results of the initial and followup surveys were largely consistent, but several institutions reported some degree of change in PF. The most striking change involved an increase in PF at one institution after initiating automatic BRAF reflex testing shortly after the initial survey. During the 6-month follow-up, this institution also noted an increase in physician referrals, which institutional representatives attributed to two factors: (i) consistent attendance of a GC at biweekly case conferences with treating physicians; and (ii) a high level of GC follow-up with physicians.

Although a few institutions moved either into or out of the Medium-PF group, no procedural changes were reported at the 14 other institutions during the 6-month time period. Nevertheless, several institutions had modified UTS procedures before the current study; these included a few High-PF institutions that increased involvement of GCs and one Low-PF institution that decreased involvement of GCs.

\section{Patient-Level Factors and PF}

All key informants identified at least one patient-level factor that they believe influences PF at their institution. Factors most commonly reported included patient concerns about cost or lack of insurance to cover genetic counseling and/or germline testing; lack of patient interest or failure to appreciate the importance of germ-line testing; and patients dealing with too many other issues. 
Table 4 Summary of study findings within the RE-AIM framework

\begin{tabular}{l} 
RE-AIM dimension \\
Description \\
\hline Reach \\
The absolute number, \\
proportion, and \\
representativeness \\
of CRC patients \\
screened
\end{tabular}

\section{Effectiveness}

The impact of UTS procedures on PF and other outcomes, including potential negative effects

\section{General findings, observations, or} future research considerations

Absolute number of newly diagnosed

CRC patients whose tumors were screened to determine who should be offered germ-line testing varies across institutions (Table 3)

Proportion screened is uncertain but estimated to approach $100 \%$

- PF with germ-line testing after a positive screen was highly variable, ranging from $\leq 10$ to $>85 \%$

- Institutions were grouped into three categories according to PF (High-, Medium-, and Low-PF)

- Few unexpected or negative outcomes have been encountered

- Only two institutions reported rare difficulties with reimbursement for tumor screening; others did not know or had no reimbursement issues

\section{Adoption}

The absolute number, proportion, and representativeness of institutions and staff who adopt UTS, and the resources and expertise available to them
- Previous studies suggest that academic institutions are more likely to adopt UTS

- GCs usually raised the idea for UTS, but multiple stakeholders were involved in the decision to adopt

- Common reasons for UTS adoption were to improve identification of Lynch syndrome patients and to benefit their family members

- Cost was a key characteristic considered in the decision to adopt UTS

Implementation

Time and costs of UTS programs, and what adaptations are made to UTS in various settings

\section{High-PF institutions}

No consistent patterns related to

PF were discerned

PF score $>40 \%$

Two High-PF institutions reported past concerns that physicians were not consistently disclosing screen-positive results or referring patients; this prompted changes to procedures that were made before the current study

- All but one of the High-PF institutions were academicresearch institutions

- The only nonacademic High-PF institution reported a greater number of challenges than facilitators to UTS adoption
Low-PF institutions

No consistent patterns related to PF were discerned

PF score $\leq 10 \%$

All Low-PF institutions were concerned about difficulties with PF

Additional concerns:

- Reflex tests may be interpreted incorrectly or not seen on pathology addendum

- Liability risks from failure to disclose screenpositive results

- Two of the five Low-PF institutions were academic institutions

- All nonacademic Low-PF institutions reported a greater number of challenges than facilitators to UTS adoption
- Substantial heterogeneity in UTS implementation exists across institutions

- Several differences are NOT consistently associated with PF (i.e., method of screening $\mathrm{IHC}$ versus MSI, and whether results are disclosed by phone or in person)

- High- and Low-PF were consistently associated with specific combinations of implementation conditions based on QCA

\section{QCA revealed that High-PF}

institutions have all of the following unique conditions:

(i) Automatic reflex testing (i.e., BRAF) is performed on a subset of screen-positive tumors

(ii) GC discloses positive results

(iii) Contacting patients is NOT a major barrier

(iv) Obtaining a referral from physician is NOT a barrier

Several adaptations have been made to overcome barriers:

- Lack of referral overcome by GC reminding physician and assisting with the referral

- Meeting patients at a follow-up appointment helped overcome contact barrier

Maintenance
Extent to which UTS
becomes part of
routine practice and
the effects of UTS
over time

- Some institutions have modified their laboratory procedures (e.g., making BRAF or hypermethylation testing automatic for a subset of tumors)

- Institutions have also changed their follow-up procedures over time (e.g., increased involvement of GCs)
- At least three High-PF institutions have changed their procedures to streamline the process and increase the involvement of GCs; these changes reportedly increased PF

\section{QCA revealed that Low-PF institutions}

have either of the following unique sets of conditions:

GC does NOT disclose results AND adoption challenges $\geq$ facilitators

OR

GC does NOT receive screening results Difficulties reported in overcoming barriers to improve PF:

- Resistance among physicians to direct patient contact by GC, or HIPAA concerns

- Too many physicians to build rapport with and educate about importance of GC and testing

- Not logistically feasible for GCs to meet patients at follow-up appointments (e.g., lack of GC personnel, geographic distance of appointments)

- One Low-PF institution used to have a GC phone patients with results and reported higher PF before changing procedures so that the $\mathrm{GC}$ no longer received screening results

CRC, colorectal cancer; GC, a master's degree-trained genetic counselor or a nurse with extensive genetic counseling experience; HIPAA, Health Insurance Portability and Accountability Act; IHC, immunohistochemical testing; MSI, microsatellite instability testing; PF, patient follow-through; QCA, qualitative comparative analysis; RE-AIM, Reach, Effectiveness, Adoption, Implementation, Maintenance; UTS, universal tumor screening. 


\section{DISCUSSION}

To our knowledge, this is the first study to systematically compare UTS outcomes across multiple institutions. Our study adds to limited outcomes data previously reported by only a few academic institutions ${ }^{33-36}$ and provides evidence that UTS programs can be successful, despite room for improvement at several participating institutions. Consistent with earlier survey research, ${ }^{17,18}$ we found substantial variability in the implementation of tumor screening across different institutions. Advancing this understanding further, we provided evidence from 15 institutions to support how variability in UTS procedures may influence PF with germ-line testing among screen-positive patients. Specifically, our proposed model suggests that higher PF can be achieved when the following conditions are implemented: (i) streamlined UTS procedures (e.g., automatic reflex to BRAF or hypermethylation, and elimination of the requirement for physician referral to genetics professionals); (ii) a high level of involvement of GCs in various UTS procedures (e.g., tracking screening results, facilitating physician referrals, following up with and/or communicating with treating physicians, and directly disclosing positive tumor-screening results to patients); and (iii) methods for overcoming barriers to patient contact and for facilitating follow-up (e.g., meeting patients at postoperative appointments).

Although this study was not designed to prove causality, plausible mechanisms exist to explain how or why key implementation conditions could improve or reduce PF. More specifically, implementing automatic reflex testing (i.e., BRAF or hypermethylation) on a subset of patients with screen-positive tumors eliminates the need to order this testing on a case-bycase basis and reduces the need for follow-up among patients who are not likely to have Lynch syndrome. In addition, requiring a physician referral creates complexity and causes PF to be contingent on (i) multiple different health-care providers' knowledge about the importance of genetic counseling and germ-line testing and (ii) their actions to both convey this to the patient and to complete a referral. Elimination of the need for referral altogether occurred only at institutions where GCs disclose positive screening results to patients. This latter condition could improve PF because GCs focus on hereditary cancer, whereas physicians have many other competing demands that may interfere with time needed to discuss results with patients. In addition, direct patient contact allows the GC to build rapport with patients early in the process and to convey to patients the importance of follow-up testing. Nevertheless, even if a GC discloses positive screening results, PF is logically contingent on successfully contacting patients. The presence of a higher ratio of adoption challenges to facilitators was a condition that helped distinguish nonacademic Low-PF institutions from Medium-PF institutions. This difference may be indicative of several organizational challenges that could reduce $\mathrm{PF}$ (e.g., communication barriers within the institution and lack of knowledge regarding the importance of PF among physicians). Nevertheless, challenges to UTS adoption are insufficient to prevent institutions from eventually achieving relatively high
$\mathrm{PF}$, as evidenced by two participating institutions in the current study.

The complexity in implementation uncovered in this study helps to explain a discrepancy among the limited data previously reported on outcomes of Lynch syndrome tumor screening. Two institutions have independently documented increases in PF after implementing a high level of involvement of GCs in follow-up and results disclosure to patients. ${ }^{33,34}$ By contrast, results from a large national survey of cancer GCs found no association between who discloses screen-positive results to patients (i.e., GCs versus physicians) and problems with $\mathrm{PF}^{18}$ In our study, disclosure of screen-positive results by GCs was insufficient for High-PF. Nevertheless, a high level of GC involvement was part of a more complex "recipe" for attaining High-PF.

Although not the focus of the current study, informants identified several patient-level factors (such as insurance coverage) that were barriers to PF. Differences in patient populations may help to explain why certain institutions have reduced PF. For example, one Medium-PF institution is located in a socioeconomically disadvantaged urban area in which sometimes patients reportedly do not even attend their postoperative appointments. Therefore, even if GCs were available to meet patients at these appointments, patient contact and PF might continue to be problematic.

Strengths of the current study include the use of methodologies for improving data credibility, reliability, and validity. ${ }^{37,38}$ First, follow-up data were gathered from 12 of the 15 key informants $\sim 6$ months after the initial survey. In addition, summaries were shared with informants and reviewed for accuracy by 11 of 15 informants. Nearly all informants reported having good tracking systems in place and were quite confident in the accuracy of the PF numbers they reported. Nevertheless, measurement limitations included self-reported data by institutional informants, the inability to collect uniform patient-level data, artificial division of institutions into three PF groups, fluctuations in PF over time, dichotomously measured conditions, and inability to prove causality. More specifically, responses from key informants (who were all GCs) could be inaccurate or biased in their favor. However, we have no reason to suspect this for the following two reasons: (i) key informants were simply asked about follow-up procedures in terms of who does what and how, rather than asking questions about whether they thought involvement of GCs improved PF; and (ii) the importance of GC involvement was confirmed when secondary informants (who were not GCs) from institutions with high PF were asked an open-ended interview question about what they believe contributes to the success of their institutions.

Thus, despite these limitations, open-ended survey responses and interview data support the proposed model. Furthermore, previous reports from two institutions document improvements in PF after making changes to UTS procedures, including implementation of BRAF reflex testing and/or increased involvement of GCs. ${ }^{33,34}$ 
As with other multiple-case studies, our model can serve as a mechanism for generalizing results. ${ }^{38}$ Nevertheless, institutions participating in our study are not representative of all cancer centers and hospitals that treat CRC patients. Furthermore, we do not know how the participating institutions compare with the 15 nonparticipating LSSN institutions that were also performing UTS at the time of the study. Consequently, additional paths to improve PF may need to be identified or forged, particularly for institutions that do not employ GCs or are unable to implement conditions that were found to be associated with high PF in our study.

Although the current study advances our understanding of UTS, we were unable to comprehensively measure all RE-AIM dimensions. The application of RE-AIM in future studies and surveillance efforts should contribute to a more complete assessment of the health impact of tumor screening to identify Lynch syndrome. Specifically, demographic data should be collected on all individuals screened, and future efforts should take into account the reduction in patient reach at those institutions wherein tumor screening is limited to a subset of patients. Additional measures of effectiveness should include the proportion of family members who are subsequently identified as a result of tumor-screening programs. This is important because cascade testing and prevention of cancers in family members determine a large portion of the public health and cost benefits of tumor-screening programs. ${ }^{22-24,39}$

Widespread adoption of routine tumor screening is critical because adoption influences the absolute number of patients who are ultimately reached. The current study focused on a relatively small number of institutions wherein GCs were involved in the adoption and implementation of UTS. Therefore, future research will need to (i) identify whether institutions that do not employ a GC have adopted UTS and (ii) characterize how UTS implementation differs at these institutions. Given that academic/research institutions appear to have been quicker or more likely to adopt UTS, ${ }^{17,18}$ health disparities could increase in rural areas or among minority populations unless UTS becomes more widely adopted. Our findings suggest that nonacademic institutions may face a greater number of challenges and/or have fewer resources or expertise needed to facilitate UTS adoption. Given the many complexities associated with UTS adoption and implementation, institutions may find the freely available resources on the LSSN website (http://www. lynchscreening.net) helpful. In addition, LSSN members can access the active listserv to troubleshoot challenging cases or seek advice from members with expertise in Lynch syndrome.

In conclusion, the results of this study are expected to help inform decision making by stakeholders and to guide future research that is needed to assess the public health impact of Lynch syndrome tumor-screening programs. The current study provides compelling evidence that tumor-screening implementation influences PF. Our model, which illustrates procedures that are expected to maximize PF, should be tested among a broader set of institutions that have implemented routine tumor screening. This model will probably need to be revised as additional methods of improving the effectiveness of tumor screening are identified. Ultimately, a larger-scale, multiinstitution study is needed to evaluate patient-level factors and to determine the relative influence of patient- versus systemlevel factors on the effectiveness of tumor-screening programs. In addition, this study highlights the importance of assessing system-level implementation conditions when future genomic technologies are integrated into health-care settings.

\section{SUPPLEMENTARY MATERIAL}

Supplementary material is linked to the online version of the paper at http://www.nature.com/gim

\section{ACKNOWLEDGMENTS}

We thank the informants who participated in this study. We also thank the Lynch Syndrome Screening Network (LSSN) for making this research feasible and for allowing recruitment through the LSSN listserv. This work was funded in part by Student Research Scholarships from the University of South Florida (USF) College of Public Health and from the Department of Community and Family Health at USF. Support for D.C.'s time was provided in part by a National Cancer Institute R25T training grant awarded to Moffitt Cancer Center (5R25CA147832-04).

\section{DISCLOSURE}

The authors declare no conflict of interest.

\section{REFERENCES}

1. American Cancer Society. Colorectal cancer facts \& figures 2011-2013. http:// www.cancer.org/Research/CancerFactsFigures/ColorectalCancerFactsFigures/ colorectal-cancer-facts-figures-2011-2013-page. Accessed 20 July 2013.

2. Hampel H, Frankel WL, Martin E, et al. Feasibility of screening for Lynch syndrome among patients with colorectal cancer. J Clin Oncol 2008;26:57835788

3. Stoffel E, Mukherjee B, Raymond VM, et al. Calculation of risk of colorectal and endometrial cancer among patients with Lynch syndrome. Gastroenterology 2009;137:1621-1627.

4. Barrow E, Alduaij W, Robinson L, et al. Colorectal cancer in HNPCC: cumulative lifetime incidence, survival and tumour distribution. A report of 121 families with proven mutations. Clin Genet 2008;74:233-242.

5. Hampel H, Stephens JA, Pukkala E, et al. Cancer risk in hereditary nonpolyposis colorectal cancer syndrome: later age of onset. Gastroenterology 2005; 129:415-421.

6. Barrow E, Robinson L, Alduaij W, et al. Cumulative lifetime incidence of extracolonic cancers in Lynch syndrome: a report of 121 families with proven mutations. Clin Genet 2009;75:141-149.

7. Watson $\mathrm{P}$, Vasen HF, Mecklin JP, et al. The risk of extra-colonic, extra-endometrial cancer in the Lynch syndrome. Int J Cancer 2008;123:444-449.

8. Järvinen HJ, Aarnio M, Mustonen $\mathrm{H}$, et al. Controlled 15-year trial on screening for colorectal cancer in families with hereditary nonpolyposis colorectal cancer. Gastroenterology 2000;118:829-834.

9. Järvinen HJ, Renkonen-Sinisalo L, Aktán-Collán K, Peltomäki P, Aaltonen LA, Mecklin JP. Ten years after mutation testing for Lynch syndrome: cancer incidence and outcome in mutation-positive and mutation-negative family members. J Clin Oncol 2009;27:4793-4797.

10. Cross DS, Rahm AK, Kauffman TL, et al. Underutilization of Lynch syndrome screening in a multisite study of patients with colorectal cancer. Genet Med 2013;15:933-940.

11. Colorectal cancer family history and genetic testing. http://www.michigan gov/documents/mdch/MIBRFSS_Surveillance_Brief_Jul_2012_Vol6No3_ FINAL_393196_7.pdf. Accessed 20 July 2013.

12. Pérez-Carbonell L, Ruiz-Ponte C, Guarinos C, et al. Comparison between universal molecular screening for Lynch syndrome and revised Bethesda 
guidelines in a large population-based cohort of patients with colorectal cancer. Gut 2012;61:865-872.

13. Morrison J, Bronner M, Leach BH, Downs-Kelly E, Goldblum JR, Liu X. Lynch syndrome screening in newly diagnosed colorectal cancer in general pathology practice: from the revised Bethesda guidelines to a universal approach. Scand J Gastroenterol 2011;46:1340-1348.

14. Moreira L, Balaguer F, Lindor N, et al.; EPICOLON Consortium. Identification of Lynch syndrome among patients with colorectal cancer. JAMA 2012;308:15551565.

15. van Lier MG, Leenen CH, Wagner A, et al.; LIMO Study Group. Yield of routine molecular analyses in colorectal cancer patients $=70$ years to detect underlying Lynch syndrome. J Pathol 2012;226:764-774.

16. Gudgeon JM, Belnap TW, Williams JL, Williams MS. Impact of age cutoffs on a lynch syndrome screening program. J Oncol Pract 2013;9:175-179.

17. Beamer LC, Grant ML, Espenschied CR, et al. Reflex immunohistochemistry and microsatellite instability testing of colorectal tumors for Lynch syndrome among US cancer programs and follow-up of abnormal results. I Clin Oncol 2012;30:1058-1063.

18. Cohen SA. Current Lynch syndrome tumor screening practices: a survey of genetic counselors. J Genet Counsel 2014;23:38-47.

19. Evaluation of Genomic Applications in Practice and Prevention (EGAPP) Working Group. Recommendations from the EGAPP Working Group: genetic testing strategies in newly diagnosed individuals with colorectal cancer aimed at reducing morbidity and mortality from Lynch syndrome in relatives. Genet Med 2009;11:35-41.

20. Palomaki GE, McClain MR, Melillo S, Hampel HL, Thibodeau SN. EGAPP supplementary evidence review: DNA testing strategies aimed at reducing morbidity and mortality from Lynch syndrome. Genet Med 2009;11:42-65.

21. Khoury MJ, Bowen MS, Burke W, et al. Current priorities for public health practice in addressing the role of human genomics in improving population health. Am J Prev Med 2011;40:486-493.

22. Ladabaum U, Wang G, Terdiman J, et al. Strategies to identify the Lynch syndrome among patients with colorectal cancer: a cost-effectiveness analysis. Ann Intern Med 2011;155:69-79.

23. Mvundura M, Grosse SD, Hampel H, Palomaki GE. The cost-effectiveness of genetic testing strategies for Lynch syndrome among newly diagnosed patients with colorectal cancer. Genet Med 2010;12:93-104.

24. Gudgeon JM, Williams JL, Burt RW, Samowitz WS, Snow GL, Williams MS. Lynch syndrome screening implementation: business analysis by a healthcare system. Am J Manag Care 2011;17:e288-e300.
25. Gausachs M, Mur P, Corral J, et al. MLH1 promoter hypermethylation in the analytical algorithm of Lynch syndrome: a cost-effectiveness study. Eur J Hum Genet 2012;20:762-768.

26. DCCPS. Cancer control research: implementation science: RE-AIM. http://www. re-aim.org/. Accessed 20 July 2013.

27. Glasgow RE, Klesges LM, Dzewaltowski DA, Estabrooks PA, Vogt TM. Evaluating the impact of health promotion programs: using the RE-AIM framework to form summary measures for decision making involving complex issues. Health Educ Res 2006;21:688-694.

28. Glasgow RE, Vogt TM, Boles SM. Evaluating the public health impact of health promotion interventions: the RE-AIM framework. Am J Public Health 1999;89:1322-1327.

29. Damschroder LJ, Aron DC, Keith RE, Kirsh SR, Alexander JA, Lowery JC. Fostering implementation of health services research findings into practice: a consolidated framework for advancing implementation science. Implement Sci 2009:4:50.

30. Ragin CC. The Comparative Method: Moving Beyond Qualitative and Quantitative Strategies. University of California Press: Berkeley, CA, 1989.

31. Rihoux B, Ragin CC. Configurational Comparative Methods: Qualitative Comparative Analysis (QCA) and Related Techniques. Sage: Thousand Oaks, CA, 2009.

32. Ragin C, Drass KA, Davey S. Fuzzy-Set/Qualitative Comparative Analysis 2.0. 2006. http://www.fsqca.com. Accessed 12 January 2012.

33. Hampel H, Lattimer I, Frankel WL. Universal Lynch syndrome screening result notification [abstract]. J Genet Counsel 2012;21:888-889.

34. Heald B, Plesec T, Liu X, et al. Implementation of universal microsatellite instability and immunohistochemistry screening for diagnosing lynch syndrome in a large academic medical center. J Clin Oncol 2013;31:1336-1340.

35. Lynch PM. How helpful is age at colorectal cancer onset in finding HNPCC? Dis Colon Rectum 2011;54:515-517.

36. South CD, Yearsley M, Martin E, Arnold M, Frankel W, Hampel H. Immunohistochemistry staining for the mismatch repair proteins in the clinical care of patients with colorectal cancer. Genet Med 2009;11:812-817.

37. Baxter $S$, Jack $S$. Qualitative case study methodology: study design and implementation for novice researchers. The Qualitative Report 2008;13:544559.

38. Yin RK. Case Study Research: Design and Methods, 4th edn. Sage Publications: London, UK, 2008.

39. Bellcross CA, Bedrosian SR, Daniels E, et al. Implementing screening for Lynch syndrome among patients with newly diagnosed colorectal cancer: summary of a public health/clinical collaborative meeting. Genet Med 2012;14:152-162. 